\title{
Peter Knopp
}

\section{Bauchreden - das Spiel mit dem Sprechen}

\section{Einleitung}

Bauchreden hat eine lange Tradition und wird bereits aus der antiken Welt berichtet. Es wird vermutet, dass Orakel und Priester diese Form der Sprachproduktion nutzten, um Äußerungen zu generieren, die Göttern und Geistern zugeschrieben werden sollten. In den Schriften von Hippocrates, Plato und Plutarch finden sich bereits Hinweise auf diese als Gastromantie bezeichnete Anwendung des Bauchredens (Vox 1993: 18-21). Andere Quellen beobachten Vergleichbares in Kulturen der Inuit, Zulu sowie in Guinea und auf den Fidschi-Inseln. Im Mittelalter wird Bauchreden oft mit Hexerei und Besessenheit in Verbindung gebracht. Ab dem 18. Jahrhundert entwickelt sich dann das Bauchreden als Unterhaltungsform und wird in zunehmend größeren Rahmen dargeboten. Heute sind Bauchredner wie Sascha Grammel, Benjamin Tomkins oder Jörg Jará regelmäßig im Fernsehen (z. B. Quatsch Comedy Club, SWR Spätschicht, NDR Intensiv Station), auf ihren eigenen Tourneen und im Internet zu sehen.

Frühe Formen des kabarettistischen Bauchredens arbeiten dabei ohne Puppen und schaffen die Illusion eines weiteren Sprechers an entfernten und „unsichtbaren“ Orten. Die vermeintlichen Sprecher waren z. B. der Schornsteinfeger im Kamin oder eine SprecherIn im Publikum, unter der Bühne oder in einer Kiste. Diese heute wenig verbreitete Form wird als distant voice ventriloquism bezeichnet (Vox 1993).

Heutzutage ist der Ort der vorgeblichen Sprachproduktion in der Regel eine Handpuppe, die von der BauchrednerIn gesteuert wird und mit der sie interagiert. Diese Variante des Bauchredens wird als near ventriloquism bezeichnet (Vox 1993). Beiden Formen ist gemein, dass die BauchrednerInnenstimme ein deutlich anderes sprechsprachliches Profil aufweisen muss als die SprecherIn, um die Illusion mehrerer SprecherInnen zu erzeugen. ${ }^{1}$

1 Da auch heute die Stimme der Puppe gelegentlich aus einer Kiste oder von unter einer Decke erscheinen soll, müssen auch zeitgenössische BauchrednerInnen in der Lage sein, den Stimmklang gemäß der von den HörerInnen erwarteten Akustik einer solchen Situation anzupassen. 


\section{Was ist Bauchreden? Stimmlich-sprachliche Strategien}

\subsection{Artikulation}

Unabhängig davon, woher die von der BauchrednerIn erzeugte Sprache vorgeblich kommt, soll die KünstlerIn selbst nicht als Quelle dieser sprechsprachlichen Äußerungen wahrgenommen werden. Ein Kennzeichen einer guten BauchrednerIn ist daher das vollständige Ausbleiben von Lippenbewegungen. Die KünstlerInnen nehmen dazu eine leicht geöffnete, oft leicht lächelnde artikulatorische Grundstellung ein, die es erlaubt, dass der produzierte Sprachschall durch den Mund entweichen kann, ohne dass die Lippen erkennbar bewegt werden.

Aus dieser leicht geöffneten Kieferposition mit leichter Spreizung der Lippen ergibt sich, dass vor allem diejenigen Sprachlaute problematisch sind, die in der modalen Sprechweise von außen gut sichtbare Bewegungen der Artikulatoren mit sich bringen. Dies sind vor allem offene Vokale wie /æ/ (engl. bad, /a/ (dt. acht) und /a/ (engl. calm), bei denen der Kieferwinkel am größten ist. Allerdings stellen auch geschlossene Vokale wie /y/ (dt. müde) und /u/ (dt. Mut) mit geringem Kieferwinkel ein Problem dar, sofern sie eine Rundung und ggf. ein Vorstülpen der Lippen erfordern. Gleiches gilt für die halboffenen gerundeten Vokale /œ/ (dt. Löss), /J/ (dt. Post) und die halbgeschlossenen gerundeten Vokale /ø/ (dt. Öl) und /o/ (dt. hoch). Westbury und Weiss (2003) weisen darauf hin, dass die leicht lächelnde Stellung der Lippen zu einer Verkürzung des Vokaltrakts (Mund-, Nasen- und Rachenraum) führt. Dies manifestiert sich im akustischen Sprachsignal in einem Absinken der für die Klangqualität der Vokale wichtigen Resonanzfrequenzen (Formanten).

Um diese Einschränkung bei der Produktion der Sprachlaute (Artikulation) zu kompensieren, muss die BauchrednerIn ihren Vokaltrakt so manipulieren, dass ein gleicher oder zumindest ähnlicher Höreindruck entsteht wie bei dem intendierten Laut. Vox (1993) beschreibt ein Anheben und Zurückziehen des Zungenrückens in Richtung Velum: „In this position, the tone is deflected so that it passes mainly through the nose cavity and partially through the mouth." (Vox 1993: 186). Wenn dabei das Velum geringfügig abgesenkt wird, entweicht ein Teil des Schalls durch den Nasenraum und ergibt den als ventriloquist drone bezeichneten hypernasalen Stimmklang.

Huizinga (1930) beobachtet anhand von Röntgenaufnahmen eine Engebildung im Pharynxbereich des Bauchredners. Dies erscheint insofern plausibel, als bei vielen aktiven BauchrednerInnen wie z. B. Sascha Grammel (Bayerischer Rundfunk 2016) oder besonders bei Jeff Dunham (2011) eine starke muskuläre 
Aktivität im oberen Halsbereich von außen sichtbar ist, die auf kompensatorische Artikulation in diesem Bereich schließen lässt. Es liegt in der Tat nahe, kompensatorische Mechanismen in Form einer Enge- oder Weitenbildung im Pharynx anzunehmen. Dies ist jedoch ein offenes Forschungsfeld - mit Ausnahme der individuellen Beobachtungen von Huizinga (1930) existieren keine empirischen Untersuchungen. Es scheint somit mindestens zwei verschiedene Strategien der Kompensation der Vokalartikulation zu geben: Die Rückverlagerung der Zunge und die Engbildung im Rachen. Dieses Prinzip ist nicht auf das Bauchreden begrenzt, sondern wird unter dem Stichwort motor equivalence auch in normaler Sprache beobachtet (vgl. Perkell et al. 1993). Das Bauchreden führt diese Prozesse aber in die Extreme des physiologisch Möglichen.

Bei den Konsonanten sind besonders die (bi)labialen /p/ (dt. Post), /b/ (dt. Bus), /m/ (dt. Mut), labio-dentalen /f/ (dt. Fisch), /v/ (dt. Wein) und labio-velaren /w/ (engl. work) Laute mit sichtbarer Lippenbewegung verbunden und für die BauchrednerIn somit problematisch. Für all diese Laute muss die BauchrednerIn daher eine optisch möglichst unauffällige kompensatorische Artikulation substituieren.

In Tabelle 1 sind die in Selbstlernbüchern für das Bauchreden (King 1997; Schindler und Tricomi 2011) üblicherweise empfohlenen Ersatzartikulationen zusammengefasst. Ihnen allen ist gemein, dass die Artikulation nach hinten und somit weiter in den Mundraum verlagert wird. Die Artikulationsart und Stimmbeteiligung bleiben gleich (stimmhafter Plosiv /b/ zu stimmhaftem Plosiv /d/), die Artikulationsstelle wird jedoch verlagert (stimmloser labiodentaler Frikativ /f/ zu stimmlosem dentalen Frikativ / $\theta /$ ).

Tab. 1: Empfohlene Ersatzartikulation bei Konsonanten

\begin{tabular}{ll}
\hline Zu ersetzender Laut & Ersatzartikulation \\
\hline$/ \mathrm{p} /$ & $/ \mathrm{t} /, / \mathrm{k} /$ \\
$\mathrm{h} /$ & $/ \mathrm{d} /, / \mathrm{g} /$ \\
$/ \mathrm{f} /$ & $/ \theta /$ \\
$\mathrm{/v} /$ & $/ \mathrm{\partial} /$ \\
$/ \mathrm{m} /$ & $/ \mathrm{n} /, / \mathrm{g} /$ \\
$/ \mathrm{w} /$ & $/ \mathrm{u} /($ entrundetes $/ \mathrm{u} /), / \mathrm{l}$ \\
\hline
\end{tabular}

Westbury und Weiss (2003) beobachten zwei grundsätzliche Strategien der Ersatzartikulation bei Konsonanten. Eine arbeitet mit geringer Variation der Artikulation und verwendet die aus Tabelle $1 \mathrm{zu}$ entnehmenden Ersatzlaute. Die 
Konsequenz ist jedoch eine hohe Abweichung im akustischen Output zwischen vorgesehenem und ersetzendem Laut. Um dem entgegenzuwirken, empfiehlt King (1997: 14): „[...] emphasizing, or stressing, the part of the word away from the difficult letter" und meint vermutlich eine Reduktion der Intensität und Dauer der Substitutionslaute, die bis zum Wegfall des entsprechenden Lautes führen kann. Gleichzeitig soll die Aussprache des restlichen Wortes betont werden. Erfahrene Bauchredner wie Ray Alan demonstrieren hingegen ihre Meisterschaft, in dem sie gerade die „schwierigen“ Laute wiederholt und betont artikulieren (vgl. morpheusatloppers 2009) und z. B. ein auditiv eindeutiges [p] erzeugen, das nicht durch einen reinen Lautaustausch mit / $\mathrm{t}$ / produziert worden sein kann.

Für diese zweite Strategie ist laut Westbury und Weiss (2003) jedoch extreme Ersatzartikulation nötig, die stark vom Modalinventar der SprecherInnen abweicht. Ähnlich der Vokalartikulation spielen hierbei vermutlich die Rachenresonanzen eine stärkere Rolle als beim normalen Sprechen. Metzner et al. (2006) können anhand eines artikulatorischen Sprachsynthesesystems ${ }^{2}$ zeigen, dass unterschiedliche Einstellungen der Artikulatoren $\mathrm{zu}$ ähnlichen perzeptiven Eindrücken führen können. Sie modellieren sowohl die natürlichen Laute /b/, /p/, /v/ und /m/ als auch je zwei alternative Konfigurationen, die so angepasst werden können, dass gute auditive Ähnlichkeit mit den natürlichen Lauten vorliegt.

\subsection{Stimmgebung und Atmung}

Da das Sprachsignal durch die bereits erwähnte geringe Mundöffnung beim Bauchreden stärker gedämpft wird als bei normaler Sprechweise, muss das im Kehlkopf gebildete Quellsignal, also der Stimmton, eine höhere Ausgangslautstärke aufweisen. Der dazu erforderliche erhöhte subglottale Druck wird durch eine stärkere Anspannung des Zwerchfells und zusätzlichen Einsatz der Bauchmuskulatur erreicht. Diese Anspannung bei einsetzender BauchrednerInnenstimme ist gut bei Jeff Dunham (2011) zu beobachten. In der Regel ist dies bei den Künstlern jedoch nicht zu sehen, da Kleidung und eine leicht gebeugte Haltung kaschierend wirken. Bauchredner wie Sascha Grammel, Jörg Jará und Peter Moreno tragen z. B. oft Sakkos, die den Blick auf den Thorax-Bereich (bewusst) versperren.

2 Die artikulatorische Sprachsynthese erzeugt Sprache mit Hilfe eines Modells des menschlichen Vokaltrakts und den artikulatorischen Vorgängen in ihm. Durch Manipulation einzelner Parameter der Artikulatoren kann das akustische Ausgangssignal verändert werden und fortlaufende Sprache synthetisiert werden. 
Huizinga (1930) stellt bei dem von ihm mittels Röntgenaufnahmen untersuchten Bauchredner allerdings eine dauerhafte Inspirationsstellung des Zwerchfells bei geringem Luftverbrauch fest. Dies erscheint jedoch wenig plausibel angesichts der Tatsache, dass die nötige Lautstärkesteigerung des Stimmtons nur durch einen höheren subglottalen Druck zu erzeugen ist. Dass mit gesteigertem Luftdruck phoniert wird, zeigt auch der von Westbury und Weiss (2003) beobachtete Anstieg der Stimmbandgrundfrequenz $\left(\mathrm{F}_{0}\right)$ einer BauchrednerIn im Vergleich zu ihrer Modalstimme. ${ }^{3}$

Die Erhöhung der Stimmlage ist eine Begleiterscheinung der größeren Stimmlippenspannung, die wiederum erforderlich ist, um dem hohen Anblasedruck der Luft standzuhalten. Durch die Spannung der Stimmlippen verringert sich die schwingende Masse, sodass - vergleichbar mit einer stärker gespannten Gitarrensaite - die Schwingungsfrequenz ansteigt.

Insgesamt sollte damit der Luftverbrauch, anders als von Huizinga beschrieben, eher ansteigen. Neue experimentelle Studien zum Luftverbrauch liegen allerdings nicht vor.

3 Dies betrifft zunächst das Bauchreden mit der Modalstimme der BauchrednerIn. Wird die Stimme wie üblich verstellt, um die Puppe stärker von der Modalstimme der KünstlerIn abzugrenzen, kann die gewählte $\mathrm{F}_{0}$ deutlich über oder unter der modalen $\mathrm{F}_{0}$ liegen. 


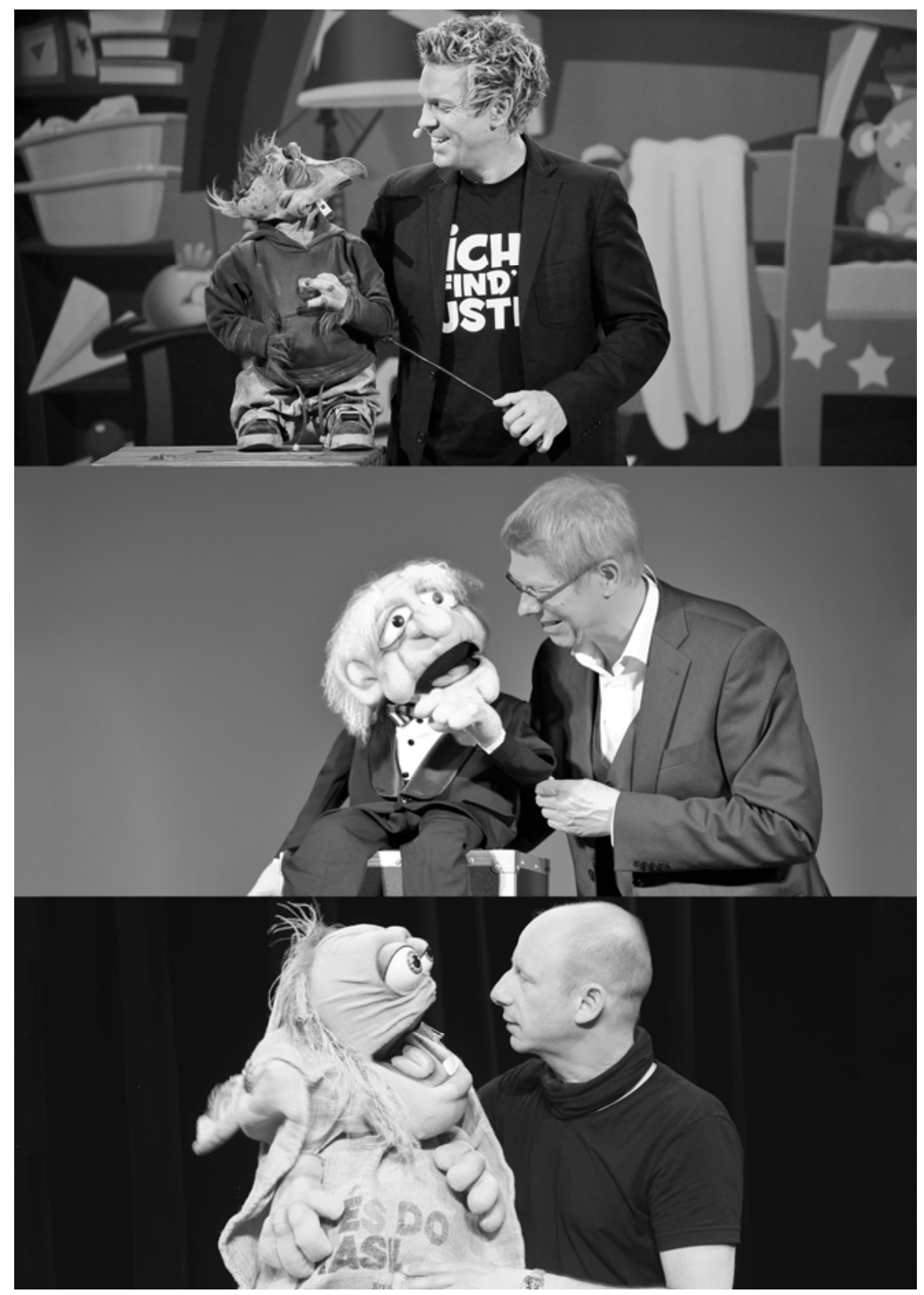

Abb. 1: Oben: Sascha Grammel (Foto: Michael Zargarinejad). Mitte: Jörg Jará (Foto: Thomas Göbert). Unten: Benjamin Tomkins (Foto: Daniele Vagt). 


\section{Nicht-sprachliche Strategien}

Neben den Kompensationen und Verschleierungen im sprachproduktorischen Bereich arbeiten BauchrednerInnen auch mit extra-linguistischen Strategien, um von sich selbst als tatsächlichen SprachproduzentInnen abzulenken und die Illusion eines zweiten Sprechers zu verstärken.

Wie eingangs bereits erwähnt, bildet beim zeitgenössischen Bauchreden die Puppe den Fokus der Illusion und Ablenkung. Die ventriloquist dolls des 19. und 20. Jahrhunderts waren überwiegend von menschlicher Gestalt und wirken auf den heutigen Betrachter eher grotesk und unheimlich. Heutzutage sind menschliche Puppen eher karikaturistisch gestaltet (z. B. Jörg Jará 2017: Herr Jensen), und es kommen oft tierähnliche Fantasiegestalten zum Einsatz (Sascha Grammel 2016: Frederic Freiherr von Furchensumpf, Benjamin Tomkins 2015: Alter Sack).

Der zentrale Effekt der Puppe ist, dass ihre Mundbewegungen synchron mit dem akustischen Sprachsignal zu sehen sind. Da die Hörwahrnehmung auch optische Reize in die Signalanalyse und -verarbeitung einbezieht, verschiebt sich die Aufmerksamkeit von der BauchrednerIn auf die „sprechende“ Puppe. Aus der Forschung zum sog. McGurk-Effekt ${ }^{4}$ ist bekannt, dass visuelle und auditive Wahrnehmung vom menschlichen Hörer integriert werden. Die Kombination von visuell dargebotener Aussprache von baba (mit den Lippen artikuliert) und auditiv dargebotener Aussprache von gaga (hinten im Mund am weichen Gaumen artikuliert) führt zum Höreindruck von dada (vorne im Mund am Zahndamm artikuliert), d. h. die beiden Sinneseindrücke werden zu einem dritten, objektiv nicht vorhandenen integriert. In ähnlicher Weise kann der weit geöffnete Mund einer Puppe den Höreindruck eines offenen /a/ verstärken, auch wenn die BauchrednerIn mit fast geschlossenem Kiefer artikuliert. Bei modernen Puppen können neben Kopf und Körperbewegungen auch Gestik durch Bein- und/oder Armbewegungen und erweiterte Mimik durch bewegliche Augen und Augenbrauen erzeugt werden. Durch diese gesteigerte Aktivität wird der Zuschauer noch stärker zur Aufmerksamkeitsverlagerung auf die Puppe angeregt, und die Illusion der belebten sprechenden Puppe wird umso glaubhafter. Die Bauchrednerin Nina Conti z. B. geht noch einen Schritt weiter, indem sie Personen aus dem Publikum pneumatische Mundmasken überzieht und ihnen über das Bauchreden Worte in den Mund legt (ukGORGEOUS 2013). Die so entstehende Diskrepanz

4 Dabei geht es um die Beeinflussung der auditiven Wahrnehmung eines akustischen Sprachsignal durch gleichzeitig beobachtete Lippenbewegungen. 
zwischen den von ihr erzeugten Aussagen und der Gestik und Mimik der beteiligten Zuschauer ist nicht nur kabarettistisch effektiv, sondern verlagert auch den Fokus der Zuschauer von ihr auf eine andere Person.

Die Interaktion mit der Puppe bietet für die BauchrednerIn weitere Möglichkeiten zur Ablenkung. Die natürliche Hinwendung zum Gesprächspartner erlaubt der KünstlerIn, ihr Gesicht teilweise vom Publikum abzuwenden und so sichtbare Bewegungen der Artikulatoren zu verbergen. Gleichzeitig wenden auch die Zuschauer ihre Aufmerksamkeit dem nun vermeintlichen Sprecher zu: der Puppe.

Zusätzlich kann die KünstlerIn bei bauchrednerisch besonders schwierig zu artikulierenden Wörtern oder Phrasen die Äußerung in Form einer unterbrechenden Nachfrage unmittelbar nach der imperfekten Äußerung der Puppe mit ihrer eigenen Modalartikulation wiederholen. Bei den Zuhörern kann dies zu einer rückwirkenden Maskierung der Puppenäußerung führen. Allzu offensichtliche Kompensationslaute können so verdeckt werden.

Auch der Charakter der Puppe kann für die Verdeckung von Kompensationsartikulation genutzt werden. Häufig genutzte Merkmale sind Puppenstimmen mit Sprachfehlern, starkem dialektalem, sozialem oder fremdsprachlichem Akzent oder auch eine von Alkohol oder Drogen beeinflusste Sprechweise. Durch das Bauchreden entstehende Abweichungen von der modalen Artikulation können so nicht mehr von denjenigen unterschieden werden, die Teil der „Rolle“ sind.

\section{Fazit}

Eine BauchrednerIn verbindet die sprachlich-kabarettistische Ebene des Sprachspiels mit einer sprachbezogenen Illusionskunst, die über die eines Puppenspielers hinausgeht, indem sie diese um eine besondere Artikulationskunst erweitert. Die Kombination aus Stimmmodifikation, Sprachproduktion ohne sichtbare Artikulation und glaubwürdiger Dialoginteraktion mit einer Puppe mit plausibler Sprech- und Gestenmotorik sorgt dafür, dass auch aufgeklärte $\mathrm{Zu}$ schauer der Illusion einer sprechenden Puppe folgen und in diesem Spiel mit dem Sprechen Unterhaltung finden.

Danksagung: Die Fotos wurden freundlicherweise von den Künstlern bzw. ihren Agenturen zum Abdruck im vorliegenden Band zur Verfügung gestellt: Panta Management GmbH (Sascha Grammel), PRIMA Künstlermanagement (Benjamin Tomkins), Jörg Jará. 


\section{Literaturangaben}

BauchrednerfanXL. 2017. Bauchredner Jörg Jará bei Karnevalissimo 2017 im ZDF. https://youtu.be/vuyTksxyims?t=2m10s (aufgerufen am 09. August 2018).

Bayerischer Rundfunk. 2016. Sascha Grammel - "Best of" Kabarett aus Franken. https://www.youtube.com/watch?v=2c2DMK1_TVQ (aufgerufen am 09. August 2018).

Dunham, Jeff. 2011. Achmed the Dead Terrorist Has a Son (Controlled Chaos): Pias Comedy. https://www.youtube.com/watch?v=IL357BrwK7c (aufgerufen am 09. August 2018).

Huizinga, Eelco. 1930. Über Bauchreden. Archiv für Ohren-, Nasen- und Kehlkopfheilkunde 127(1). 77-92.

King, Kolby. 1997. Ventriloquism made easy. Mineola, N.Y.: Dover Publications.

Metzner, Jörg, Marcel Schmittfull \& Karl Schnell. 2006. Substitute sounds for ventriloquism and speech disorders. In ISCA (Hg.), INTERSPEECH 2006 - ICSLP, Ninth International Conference on Spoken Language Processing, Pittsburgh, PA, USA, September 17-21, 2006,1379-1382. http://www.isca-speech.org/archive/interspeech\_2006/i06\_ 1426.html.

morpheusatloppers. 2009. Ray Alan with „Lord Charles“ - World's Greatest Ventriloquist 1986. https://youtu.be/C3Zn3M-WMzM?t=3m22s (aufgerufen am 09. August 2018).

Perkell, Joseph S., Melanie L. Matthies, Mario A. Svirsky \& Michael I. Jordan. 1993. Trading relations between tongue- body raising and lip rounding in production of the vowel $/ u /: A$ pilot "motor equivalence" study. The Journal of the Acoustical Society of America 93(5). 2948-2961.

Schindler, George \& Ed Tricomi. 2011. Ventriloquism: Magic with your voice. Mineola, N.Y.: Dover Publications.

Tomkins, Benjamin. 2015. Bauchredner Benjamin Tomkins Märchenstunde mit dem alten Sack. https://www.youtube.com/watch?v=uvoW02t3PVU\&t=327s (aufgerufen am 09. August 2018).

ukGORGEOUS. 2013. Nina Conti Hilarious (Strong Language). https: //www.youtube.com/watch?v=03vBYHvG2wE\&t=217s (aufgerufen am 09. August 2018).

Vox, Valentine. 1993. I can see your lips moving: The history and art of ventriloquism, Rev. enlarged ed. North Hollywood, Calif., Studio City, CA: Plato Pub.; Distributed by Empire Pub. Service.

Westbury, John R. \& Clarissa J. Weiss. 2003. Articulator movements in ventriloquists' speech. In M. J. Solé \& Daniel Recasens i Vives (Hgg.), Proceedings of the 15th International Congress of Phonetic Sciences, Barcelona 3-9 August 2003, 1037-1040. Barcelona: Universitat Autónoma de Barcelona.

Peter Knopp studierte an der Universität Trier Phonetik und Anglistik und schloss dieses Studium mit dem Magistergrad ab. Er wurde 2018, mit einer Arbeit über den Zusammenhang von Musikalität und der Fähigkeit, Sprecher anhand ihrer Stimme wiederzuerkennen, promoviert. Er unterrichtet und forscht seit $2010 \mathrm{im}$ Fach Phonetik mit den Schwerpunkten Ohrenzeugen, Physiologische Phonetik sowie alternative Sprach- und Stimmproduktion. 
